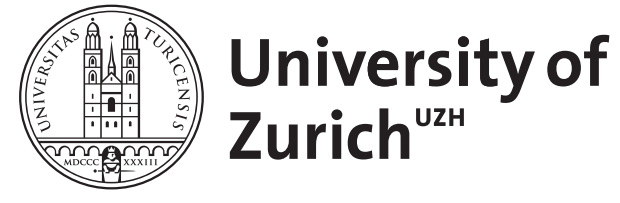

\title{
Requisitos para ser lengua: el caso del asturiano y de otras modalidades lingüísticas de España
}

Kabatek, Johannes

Posted at the Zurich Open Repository and Archive, University of Zurich ZORA URL: https://doi.org/10.5167/uzh-85914

Book Section

Originally published at:

Kabatek, Johannes (2006). Requisitos para ser lengua: el caso del asturiano y de otras modalidades lingüísticas de España. In: Kabatek, Johannes; Castillo Lluch, Mónica. Las lenguas de España. Política lingüística, sociología del lenguaje e ideología desde la Transición hasta la actualidad. Frankfurt a.M./Madrid: Veruert, 141-158. 


\title{
REQUISITOS PARA SER LENGUA: EL CASO DEL ASTURIANO Y DE OTRAS MODALIDADES LINGÜÍSTICAS DE ESPAÑA
}

\author{
JOHANNES KABATEK
}

\section{Los discursos acerca de las lenguas de España}

Los discursos acerca de las lenguas, como los discursos acerca de los objetos en general, se generan en determinadas situaciones, a partir de las cuales se vuelven en parte autónomos y adquieren su tradición propia. La autonomía de los discursos metalingüísticos permite su transformación y su aplicación en lugares o situaciones distintas, ajenos al lugar original de creación. Por mucha que sea la diferenciación interna del espacio comunicativo de España, también se pueden observar, al menos desde el siglo XVIII, redes comunicativas que permiten la migración de los discursos metalingüísticos, en nuestro caso de los discursos acerca de las modalidades lingüísticas. Son esas redes comunicativas las que generan la supuesta poligénesis del discurso de la ilustración y su postulado de respeto por las modalidades lingüísticas maternas de los diferentes pueblos dentro de España. Y son ellas las que llevan al paralelismo de los diferentes movimientos de reivindicación regional en el siglo XIX. Se podría escribir una historia de las lenguas y variedades lingüísticas de España en términos de una historia de génesis y migración de discursos, incluyendo evidentemente los discursos generados fuera de España, en Francia y Alemania, sobre todo.

Nos parece un aspecto de suma importancia, a la hora de presentar un balance de la política lingüística en España desde la Constitución de 1978, hacer hincapié en ese espacio discursivo común, con marco legal explícito en la propia Carta magna. Con todo, aunque los discursos viajen, las situaciones a las que se aplican son bien diversas, y la cuestión que hay que plantearse es cuáles son los efectos de la adopción de un discurso creado en ciertas zonas de Cataluña, por ejemplo, en el País Vasco o en Galicia. Y más allá de las tres comunidades "históricas", ¿cuál será el efecto de adopción de discursos como el de la "normalización lingüística", de la "lengua propia", de los "derechos a la lengua materna" en otras regiones, como en Aragón, en Canarias o en Asturias? Está claro que la adopción de esos discursos tiene límites, y que hace falta una mínima base histórica, lingüística y de conciencia lingüística para que esta adopción sea posible. En este artículo me limitaré básicamente al caso del asturiano, quizá el más discutido de los mencionados, ya que para algunos su situación es bien comparable 
a la del catalán, del éuscaro y más aún del vecino gallego, mientras que para otros se trata de una situación claramente distinta y sin fundamento comparable a las otras tres. Esta falta de unanimidad en la valoración de la situación lingüística asturiana deriva, como veremos, de la interpretación de los hechos históricos y actuales y no permite una decisión "objetiva" derivable del análisis sociolingüístico. El análisis únicamente puede enumerar los factores de los cuales derivan los diferentes juicios y preguntarse por las posibilidades de cada una de las tendencias. En las siguientes líneas, reflexionaremos sobre la base histórica, la base lingüística y las actitudes de los asturianos con respecto a la actual situación lingüística de Asturias.

\section{La base histórica del asturiano}

La Academia de la Llingua Asturiana da la siguiente descripción definitoria del asturiano:

L'asturianu ye una llingua románica que se fala nel Principáu d'Asturies -comunidá autónoma del noroeste d'España-, anque tamién se caltién, con mayor o menor puxu, en fasteres más aisllaes de Lleón y Zamora, arriendes d'en Miranda del Douru (Portugal), u se fala'l “mirandés”, variedá llingüística del dominiu ástur qu'apocayá tien reconocencia como idioma "oficial" nes tierres de Miranda (Academia de la Llingua, 2002).

Esta definición considera el asturiano como lengua románica en el concierto de las demás lenguas románicas. Mientras que la tradición de la filología románica o no planteaba la cuestión lengua-dialecto en sus menciones acerca del asturiano o bien lo consideraba dialecto iberorrománico y no lengua, desde hace algún tiempo van apareciendo cada vez más menciones sobre el asturiano como lengua al lado de otras lenguas románicas. Así por ejemplo, aparece el asturiano como lengua en el prestigioso Lexikon der Romanistischen Linguistik, en algunas recientes introducciones y en el catálogo Ethnologue de las lenguas del mundo ${ }^{1}$. Para algunos, queda así aclarada la cuestión de clasificación y el asturiano se ha ganado un lugar entre las lenguas romances. Aún así, no se trata de un mero problema de etiquetaje por parte de algún editor o autor determinado, sino de la cuestión del estatus del asturiano como modalidad lingüística y su estatus en la sociedad asturiana, por lo que conviene buscar más información en este sentido.

\footnotetext{
$1<\mathrm{http}: / /$ www.ethnologue.com/>.
} 
El análisis de la base histórica del asturiano exige dos comentarios previos: en primer lugar, aunque la base histórica aparezca muchas veces como argumento de apoyo en los movimientos de normalización lingüística actuales, la historia lejana solo juega un papel indirecto para la realidad actual, un papel de apoyo discursivo, pero no de hechos inmediatos. Así, por ejemplo, de poco le sirve al occitano su glorioso pasado medieval si en el presente solo pocos lo aprecian y lo hablan. Por otro lado, una lengua sin pasado literario medieval como la vasca no tiene por qué ser menos importante en la actualidad que otras que sí lo tienen. El segundo comentario se refiere al estatus de las lenguas en la Edad Media: los romances medievales, inclusive los más desarrollados como el castellano y el catalán, no eran ni lenguas uniformizadas ni lenguas "plenamente normalizadas", como a veces se lee. Todos los romances medievales existían en convivencia diglósica con el latín y carecían de una unidad comparable a la de los estándares modernos. Nuestra concepción de lengua suele ser, por lo menos en parte, una concepción moderna que se refiere más a un estándar unitario que a un conjunto de variedades; sin embargo, tal estándar no existe en la Edad Media y la idea de "la lengua medieval" con respecto a los romances es peligrosamente anacrónica.

En la Edad Media, el romance de Asturias forma parte del continuo de los dialectos del Norte de la Península a partir de los cuales, básicamente por el desarrollo territorial de la Reconquista, se van formando los espacios lingüísticos de la Península Ibérica. Entre los dialectos del norte, el romance asturiano tiene una posición privilegiada ya que es el primer romance al que corresponde una entidad estatal. De hecho es en Asturias y, posteriormente, en el reino de León donde aparecen, de forma más destacada que en otras zonas de la Península Ibérica, textos latinos de fuerte influencia romance local, el llamado y discutido "latín vulgar leonés" de Menéndez Pidal². A partir del siglo XII hay testimonios del empleo del romance local en textos jurídicos con tradiciones más o menos estables, textos de lengua mezclada como el Fuero de Avilés; otros como el Fuero de Oviedo, el Fuero de Campomanes o la traducción leonesa del Liber iudiciorum como Fueru Xulgu. Pero pronto el "hermano pequeño" del conjunto León y Castilla interrumpe el desarrollo de la lengua en Asturias y el castellano se superpone a la lengua escrita local. Más importante para el desarrollo posterior que la reducida continuidad de producción escrita a través de los siglos siguientes será, pues, como en Galicia, la continuidad del asturiano en la oralidad y la superficialidad de la castellanización fuera de ciertos ámbitos sociales (iglesia, nobleza) y locales (urbanos). A diferencia del gallego, sin embargo, la geografía de Asturias y la escasa comunicación interdialectal fomentaron la fragmentación dialectal (o

\footnotetext{
2 Menéndez Pidal (1926: 454-455); Wright (1989: 250-264).
} 
impidieron la unidad). La diglosia entre lengua escrita y dialectos locales es observada repetidas veces y criticada por intelectuales como Antón González Reguera en el siglo XVII o en el círculo de Jovellanos en el siglo XVIII. Jovellanos es considerado en la actualidad como el fundador del regionalismo asturianista ya que en parte la ilustración de Asturias también comprendía el estudio de la lengua local. Aunque el encargo que Jovellanos dio a Francisco de Paula Caveda en el marco del Instituto Asturiano fundado por Jovellanos en los años 1790 de escribir una gramática y un diccionario asturiano no se lleva a cabo, en su entorno hay una cierta actividad literaria cuyo testimonio es la colección de poesía asturiana publicada en 1839 por el hijo de Francisco de Paula Caveda, José Caveda y Nava. Son las primeras décadas del siglo XIX, sobre todo después de las invasiones francesas y en parte como consecuencia de las ideas de la Ilustración, las que producen un cierto número de ejemplos de producción literaria, sobre todo poética. Esta nueva producción literaria local se enmarca dentro del contexto del romanticismo europeo y representa en parte una reacción particularista ante las ideas uniformadoras de la Revolución Francesa ${ }^{3}$. Además, la mayor presencia de la escuela y, sobre todo a partir de la segunda mitad del siglo XIX, la inmigración obrera castellanoparlante, llevan a una mayor extensión del castellano fundamentalmente en las zonas urbanas y mineras.

Como en el caso de otros movimientos regionalistas europeos, la lengua local aparece prácticamente solo como instrumento literario, pero acompaña un regionalismo que enfoca también intereses económicos y políticos sin que el vínculo entre esa expresión y el uso de la lengua local sea directo. Si comparamos el movimiento literario asturiano de mediados del siglo XIX con el de la vecina Galicia, más exitoso a largo plazo cuando se piensa en la situación del siglo siguiente, es sorprendente ver cómo en parte avanza más el asturiano que el gallego en la fase inicial en cuanto a la consolidación de la lengua escrita y la homogeneidad de las tendencias literarias ${ }^{4}$. Un caso emblemático en este contexto es el de las traducciones gallega y asturiana del evangelio de San Mateo encargadas a mediados del siglo desde Londres por el príncipe Luis Luciano Bonaparte, sobrino de Napoleón y coleccionista de muestras de lenguas y dialectos de Europa para fines lingüísticos ${ }^{5}$. Los encargos de Bonaparte llevan, en ambos

\footnotetext{
3 Se trata aquí de una constante en la historia de las lenguas: las reformas carolingias y cluniacences, cuyo objetivo es la uniformización del latín, llevan al surgimiento de las lenguas románicas; el jacobinismo lingüístico lleva a los movimientos de reivindicación lingüística del siglo XIX; y la europeización y el uniformismo globalizante de la actualidad llevan a un mayor deseo por acentuar la particularidad.

4 Véase a este respecto Bauske (1991).

5 Cf. Kabatek (2003a).
} 
casos, a la creación del primer texto extenso en prosa desde de la Edad Media. Pero mientras que la primera traducción gallega encargada por Bonaparte es rechazada por el príncipe dado su alto grado de dependencia del castellano y su falta de criterio autóctono, en Asturias Bonaparte encuentra un traductor ideal en el personaje de Manuel Fernández de Castro (1825-1895). Fernández de Castro aprovecha el encargo para la construcción de una especie de koiné literaria, con el criterio de la representatividad de las formas elegidas y el intento de recogida amplia del léxico dialectal. En el caso del gallego, Bonaparte publica después otra versión revisada en Londres sin que en Galicia se sepa de la publicación del primer libro de prosa religiosa gallega del siglo XIX, que pudo haber servido de modelo ortográfico para los textos posteriores. En Asturias, en cambio, la versión de Fernández de Castro es conocida ${ }^{6}$ y forma así parte de la tradición literaria asturiana, poniendo a disposición de los escritores un modelo de orientación elaborado con criterios conscientes de una especie de planificación lingüística y ortográfica. Dice Fernández de Castro, en un comentario de su traducción, que "estamos dando principio ahora á la gramática asturiana" y que ha intentado solucionar el problema de la heterogeneidad dialectal eligiendo elementos de distintas hablas:

creo que recogí de todas las prov. alguna cosa, eligiendo siempre entre los distintos términos aquellos que tenian mas afinidad con la lengua madre (apud Kabatek 2003a: 34).

Evidentemente, en esto también siempre hay algo de casualidad, y por mucho que haya individuos brillantes como Fernández de Castro, su éxito siempre dependerá de la capacidad que tenga su entorno de querer dar continuidad a sus proyectos. En Galicia tenemos en la misma época con Rosalía de Castro una personalidad de gran prestigio literario aunque de criterio lingüístico más defectuoso, pero encontramos al mismo tiempo más continuidad, y el galleguismo se consolida de generación en generación. Mientras en Cataluña, el País Vasco y Galicia se van articulando y consolidando los nacionalismos locales a finales del siglo XIX con las ilustres personalidades de Enric Prat de la Riba, de Sabino Arana y de Manuel Murguía, en Asturias ni hay un nacionalismo organizado ni se construye una mitología comparable a la de los tres otros $\operatorname{casos}^{7}$. Evidentemente hay continuadores del regionalismo asturiano también en el siglo xx, con el Catecismo

\footnotetext{
${ }^{6}$ Como lo es también su versión de la Bula ineffabilis, otro texto cuya versión gallega solo se conoce en Galicia un siglo más tarde mientras que la asturiana está presente en los movimientos literarios asturianos del XIX.

7 Para lo que sigue, nos apoyaremos en el excelente resumen de Bauske (1995).
} 
regionalista de 1918 en la línea de las ideas de Prat de la Riba, o la idea de crear una Academia de la lengua. Sin embargo, el regionalismo asturiano no tiene la fuerza que tienen los otros tres, y además, se desvincula cada vez más de la cuestión lingüística. Frente a una burguesía catalana que se expresa en catalán, un nacionalismo vasco para el que la lengua, aunque poco hablada, es el máximo exponente de la milenaria particularidad de los vascos y un galleguismo que cuenta con la casi totalidad de la población que habla un gallego relativamente uniforme, en las ciudades asturianas predomina la conciencia dialectal, de una pluralidad de hablas al lado de la lengua de prestigio uniforme que predomina en las ciudades. Cuando Sabino Álvarez Gendín describe en 1932 los problemas fundamentales de Asturias desde una perspectiva regionalista ${ }^{8}$, no se menciona el asturiano ni como lengua ni como dialecto y únicamente se habla de la necesidad de "fomentar la cultura y la riqueza regional"".

Mientras en los últimos años de la dictadura franquista en las otras regiones se va preparando la fase de la llamada "normalización lingüística" y se consolidan los movimientos estudiantiles e intelectuales que mediante estudios y actividades literarias, unidas a la connotación antifranquista de las lenguas regionales, preparan el terreno de una rápida emancipación en las siguientes décadas, en Asturias se cuestiona la posibilidad de llevar a cabo un proyecto de reivindicación lingüística, y el regionalismo se concentra en problemas políticos y económicos. En el mencionado estudio de Bauske ${ }^{10}$, se cita como emblemático el artículo de Carmen Díaz Castañón publicado en 1968 bajo el título de "Literatura bable", en el que se afirma lo siguiente:

el bable, como instrumento lingüístico estructurado, ha desaparecido ya de nuestra Asturias, quizá sería lo mejor limitarse a conservar de él un acento, unas palabras, unos matices, sin empeñarnos en emplearlo como vehículo literario, cosa que realmente no fue ni en sus mejores momentos (apud Bauske 1995, 29).

Sin embargo, a partir de los años setenta, en un clima general de cambio y de establecimiento de nuevos discursos, también en Asturias se van formando grupos que proclaman la emancipación del bable y la "normalización" de una lengua asturiana. En 1969, se crea el grupo Amigos del Bable, y a partir de 1971 se publicarán semanalmente textos en bable en el periódico Región; más adelante se crea el periódico Asturias semanal y el Conceyu Bable, organización cuyo

\footnotetext{
${ }^{8}$ Regionalismo. Estudio general. El problema de Asturias, cf. Bauske (1995: 25).

9 Cit. en Bauske (1995: 26).

10 Véase nota 7.
} 
objetivo es la promoción del bable. Las actividades en pro del bable provocan toda una serie de polémicas entre los que cuestionan la posibilidad y la necesidad de elaborar una lengua asturiana que se pueda emplear en todos los ámbitos de la sociedad y los que precisamente postulan que así se haga. Los discursos de emancipación hacen eco de los discursos de otras comunidades españolas y raras veces plantean una tercera vía, de conservación de la variedad dialectal frente a la lengua estándar ya existente, el castellano. Las alternativas que parecen darse son la de una plena normalización, por un lado, o la desaparición del bable a largo plazo, por el otro.

La reactivación del tema de la lengua encuentra apoyo en la fórmula abierta del artículo 3 de la Constitución de 1978, que permite a los que así lo deseen considerar el asturiano como una de las "lenguas de España" mencionadas pero no precisadas. Aun así, en el caso del asturiano, la decisión política tomada en el Estatuto de Autonomía se queda a medio camino entre la inoficialidad anterior y la completa cooficialidad de la que gozan catalán, vasco y gallego en sus respectivos estatutos. El asturiano es mencionado y se proclama su protección, pero no es oficializado mediante el artículo 4 del Estatuto de Autonomía de 1981:

El bable gozará de protección. Se promoverá su uso, su difusión en los medios de comunicación y su enseñanza, respetando en todo caso las variantes locales y la voluntariedad en su aprendizaje.

Esta fórmula sí implica una especie de "tercera vía" y refleja el compromiso entre la posición bablista que postulaba la oficialización del asturiano siguiendo el modelo de las tres lenguas regionales cooficiales y la posición de los que seguían considerando el asturiano como conjunto dialectal sin base suficiente para una elaboración o simplemente no favorecían tal proceso por diversas razones.

\section{La situación actual}

Los 80 y 90 son años en los que la enseñanza sistemática de las lenguas regionales crea una primera generación enteramente alfabetizada en catalán, vasco o gallego; años en los que los medios de comunicación autonómicos van pasando, en diferentes grados, paulatinamente desde el choque de las primeras películas americanas dobladas en la lengua local hasta la completa cotidianidad de tal fenómeno; años, pues, de la presencia de la lengua local en prácticamente todos los ámbitos anteriormente reservados al castellano. También en Asturias son años de extensión del movimiento asturianista, años de numerosas publicaciones en asturiano y de consolidación de un asturiano urbano, marcado, como también 
el neo-gallego y el éuscaro urbano, por la presencia de neohablantes cuya lengua materna -y, en muchos casos, la de uso mayoritario- es el español. Igual que en el caso gallego, la semejanza del castellano produce numerosas interferencias e intentos por parte de los hablantes de evitarlas (los cuales también llevan a la producción de textos influenciados por la lengua de contacto) sobre todo en el caso de los textos escritos elaborados, salpicados de elementos que marcan la diferencia con respecto al tan semejante castellano.

La diferencia política entre Asturias y las comunidades en las que se logró la plena cooficialidad de una lengua local permite, en opinión de algunos, una especie de estudio "doble ciego" (double blind), de comparación de dos situaciones de inicio parecida pero de política lingüística diferente, como en opinión del sociólogo Francisco Llera Ramo, según el cual dentro de España Asturias es "la única Comunidad bilingüe sin oficialidad ni política lingüística" (2003: 326). Según su opinión, la actual situación sociolingüística de Asturias se debe a la falta de una política lingüística coherente durante las últimas décadas, frente a lo cual él postula la oficialización del asturiano. Pero la idea del estudio "doble ciego" falla en dos aspectos: por un lado, la situación inicial no es comparable a la de las otras tres comunidades y, por ello, la base para una política lingüística en favor del asturiano es mucho más débil. Y el segundo aspecto es que la voluntad del pueblo asturiano en favor de una política lingüística en pro de la lengua local desde la instauración de la democracia tampoco es comparable a la que se puede observar en las otras comunidades. No queremos decir aquí que una política lingüística coherente de cooficialidad del asturiano no hubiera podido alterar la situación asturiana en los treinta años desde la muerte de Franco, pero el hecho de que tal política no haya tenido lugar también corresponde a la falta de una voluntad decisiva en este aspecto por parte de las fuerzas políticas en Asturias y, en último término, del pueblo asturiano. El mencionado sociólogo Francisco Llera Ramo presentó, entre los años 1991 y 2003, una serie de estudios sobre el papel del asturiano en la sociedad que presentan algunos resultados muy ilustrativos de esta situación. Miremos algunos de los resultados del estudio de 2003, II Estudio sociolingüistico de Asturias ${ }^{11}$, hecho a base de encuestas representativas en las que los asturianos daban su opinión acerca de una serie de cuestiones relativas a la situación lingüística. En primer lugar, en cuanto a la cuestión de la demanda de una normalización lingüística, los datos del estudio confirman la posición de los que postulan una "normalización" lingüística ya que a la pregunta de si están a favor o no de ella, una clara mayoría contesta que sí:

\footnotetext{
${ }^{11}$ Reproducimos los gráficos con el amable permiso de la Academia de la Llingua Astu-
} riana. 


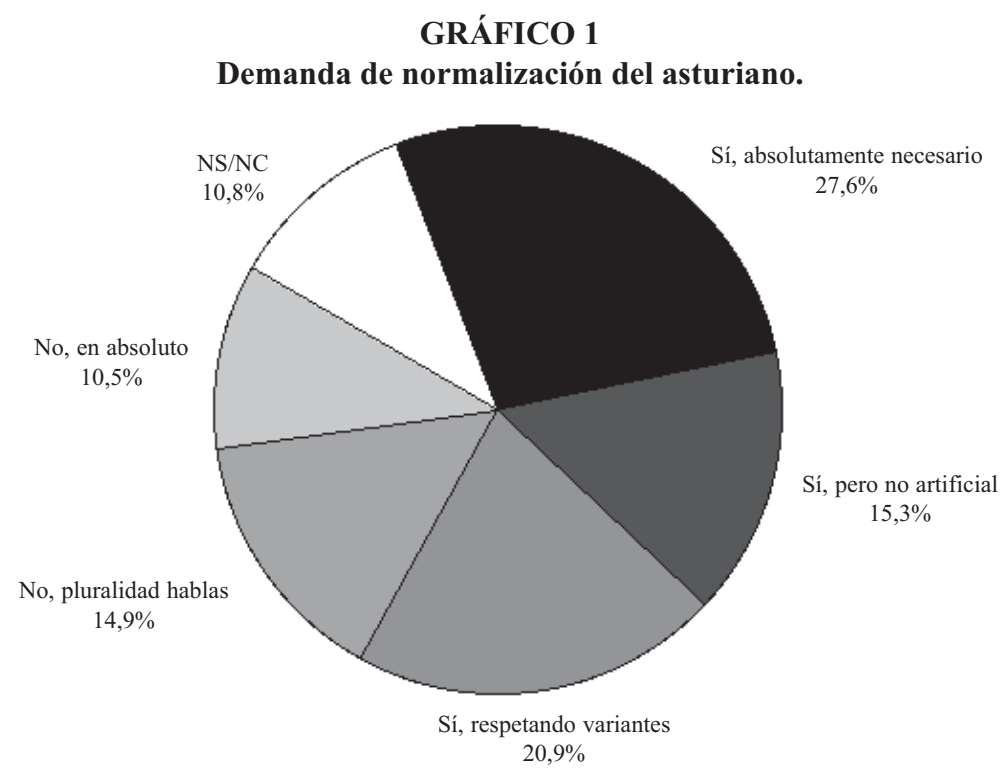

FUENTE: II Estudio sociolingüistico de Asturias - 2002 (en: Llera Ramo 2003: 158).

Este consenso básico ha aumentado, además, en los últimos años, pero en las respuestas de la encuesta se señala un problema añadido del asturiano urbano, el hecho de que sea considerado "artificial" por muchos asturianos. El fenómeno de la "artificialidad" es conocido y se da en todas las situaciones de elaboración lingüística y de introducción de una nueva lengua estándar anteriormente desconocida. La política lingüística conoce varias estrategias para combatir esa sensación, que pueden estar relacionadas tanto con la planificación del corpus como con la del estatus. En cuanto a la primera, se puede proponer, al menos provisionalmente, la aceptación de un cierto polimorfismo también a nivel del estándar, según la idea de lo que se ha llamado un "estándar polinómico", que evita un choque demasiado brusco con la nueva realidad creada. A nivel de la planificación del estatus, se puede propagar la idea de que la creación de un estándar no afecta a la realidad de la variedad hablada y que es únicamente una convención para la lengua escrita y para ciertos usos comunes. Con todo, hace falta un consenso mínimo para que el esfuerzo de elaboración de una lengua cuente con el apoyo necesario por una parte considerable de la población. La cuestión de la unidad del estándar es un problema bien conocido dentro del contexto de las lenguas de España ya que marcó, entre otras, una larga polémica en Cataluña y el debate acerca del euskara batua. En estos dos casos, el problema de la diversidad nunca puso en duda el consenso fundamental sobre la reivindicación por parte de la mayoría de una oficialización de las respectivas lenguas, cosa que no parece ser el caso en Astu- 
rias, donde la diversidad dialectal -real e imaginada- es considerada uno de los mayores obstáculos para una oficialización. Mientras que en Galicia, por ejemplo, hay una conciencia generalizada de la unidad del gallego (acompañada de una conciencia de diferencias dialectales identificables mediante una serie de marcadores pero que no ponen en cuestión la unidad fundamental), en Asturias la pluralidad de "los bables", confirmada por los dialectólogos sobre todo en las zonas de transición, pero tal vez más como un problema de percepción metalingüística que de hechos lingüísticos objetivos, hace que más de un $20 \%$ considera como diferente el dialecto local incluso con respecto a los dialectos vecinos ${ }^{12}$ :

\section{GRÁFICO 2}

\section{Parecido del habla local con otras hablas}

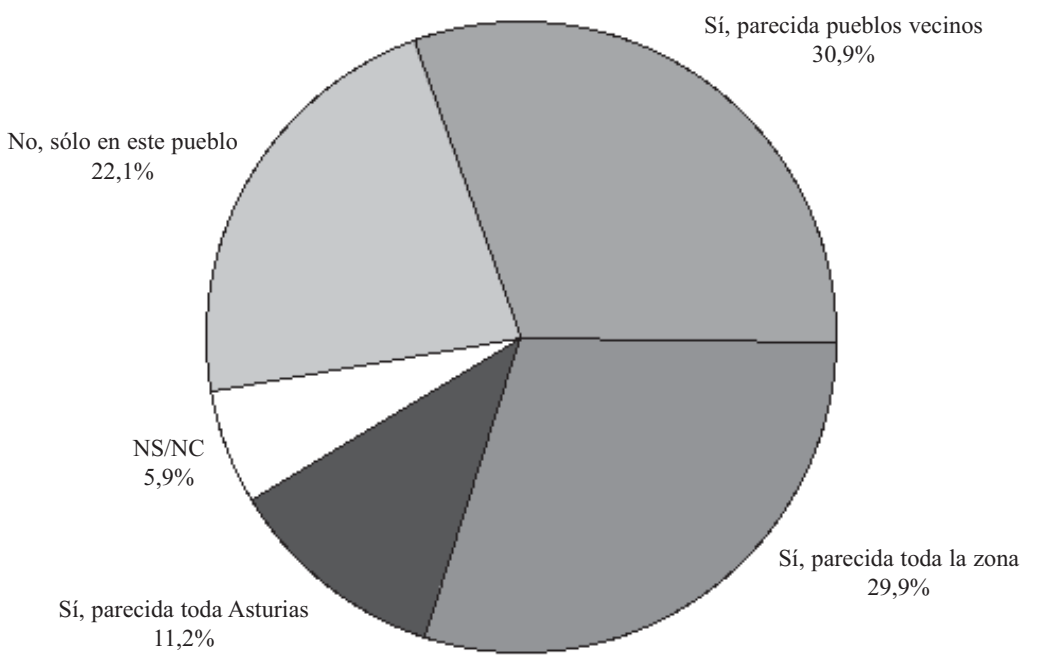

FUENTE: II Estudio sociolingüistico de Asturias - 2002 (en: Llera Ramo 2003: 122).

Con todo, el aumento de demanda de normalización del asturiano va acompañado de un aumento de su consideración como lengua y no como dialecto. Si se comparan los datos obtenidos por Llera Ramo en 1991 sobre esta cuestión con datos de 2002, se constata una clara diferencia, aunque el dato sigue siendo relativo si pensamos en el $30 \%$ que le sigue negando al asturiano el estatus de lengua.

12 Este tipo de percepción es generalmente conocido por la dialectología y parece que no es el hecho que diferencia el caso asturiano de los de las otras lenguas de España. El problema reside más bien en la falta de percepción de una unidad lingüística superior a la del dialecto local. 
GRÁFICO 3

¿Es el asturiano una lengua?

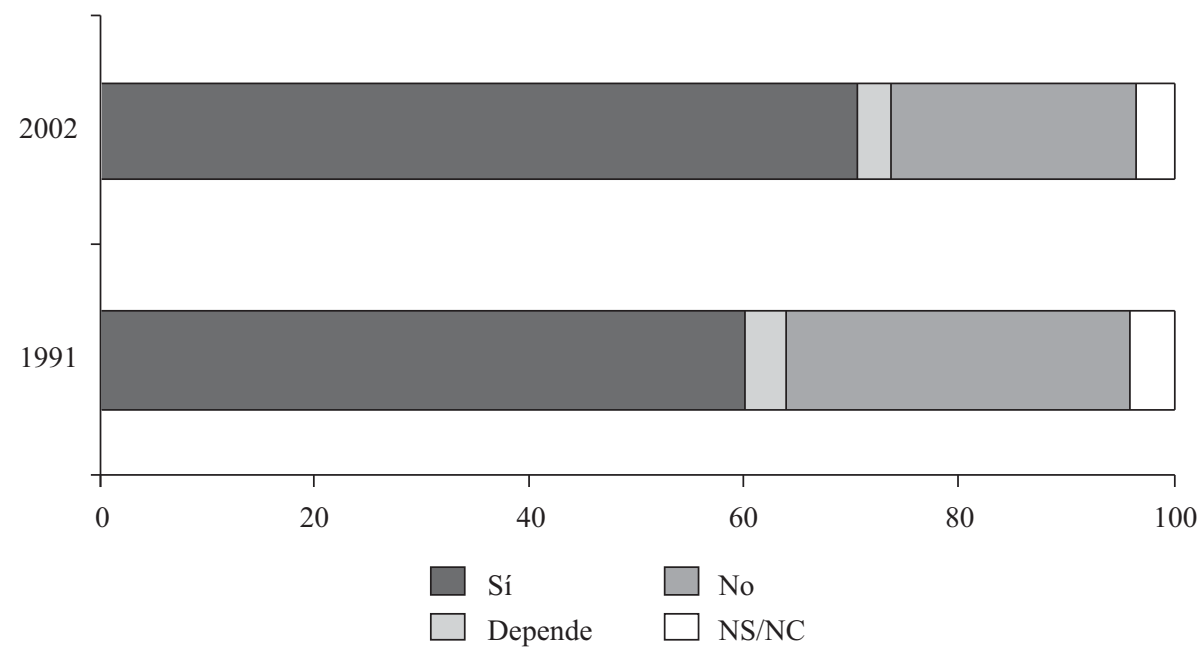

FUENTE: Llera Ramo 1991, II Estudio sociolingüistico de Asturias - 2002 (en: Llera Ramo 2003: 106).

Aquí se trata de un dato de conciencia metalingüística que posiblemente sería modificable si el poder político o instituciones prestigiosas lo propagaran. Pero al lado de la cuestión de consideración general del estatus del asturiano hay otra, mucho más difícil de cambiar, que es la propia competencia lingüística de los asturianos. A la cuestión acerca de la lengua en que con más facilidad se expresa, solo el 17,6\% (de los 49\% de asturianos que dicen que saben hablar asturiano) contesta que es el asturiano; el $43 \%$ dice expresarse con la misma facilidad en asturiano y español y casi el 34\% dice expresarse con más facilidad en español, es decir que solo el $9 \%$ de la totalidad de la población dice que se expresa con más facilidad en asturiano, mientras alrededor del $67 \%$ afirma expresarse con más facilidad en castellano ${ }^{13}$ (Gráfico 4).

Si comparamos estos datos con el nivel de conocimiento de la modalidad lingüística local que indican los habitantes de las diferentes comunidades plurilingües, Asturias se sitúa aún por encima del País Vasco y de Navarra (sin contar el

${ }^{13}$ No nos cansamos de señalar, sin embargo, que los datos aquí presentados son datos a base de respuestas a una encuesta, diferentes de los datos "reales" del comportamiento. Siempre hay que preguntarse en qué medida corresponden las creencias y actitudes expresadas en una situación particular de entrevista al comportamiento real de los hablantes. 


\section{GRÁFICO 4}

Lengua de expresión oral más fácil. Gráfico elaborado a base del $49 \%$ que dice que es capaz de expresarse en asturiano

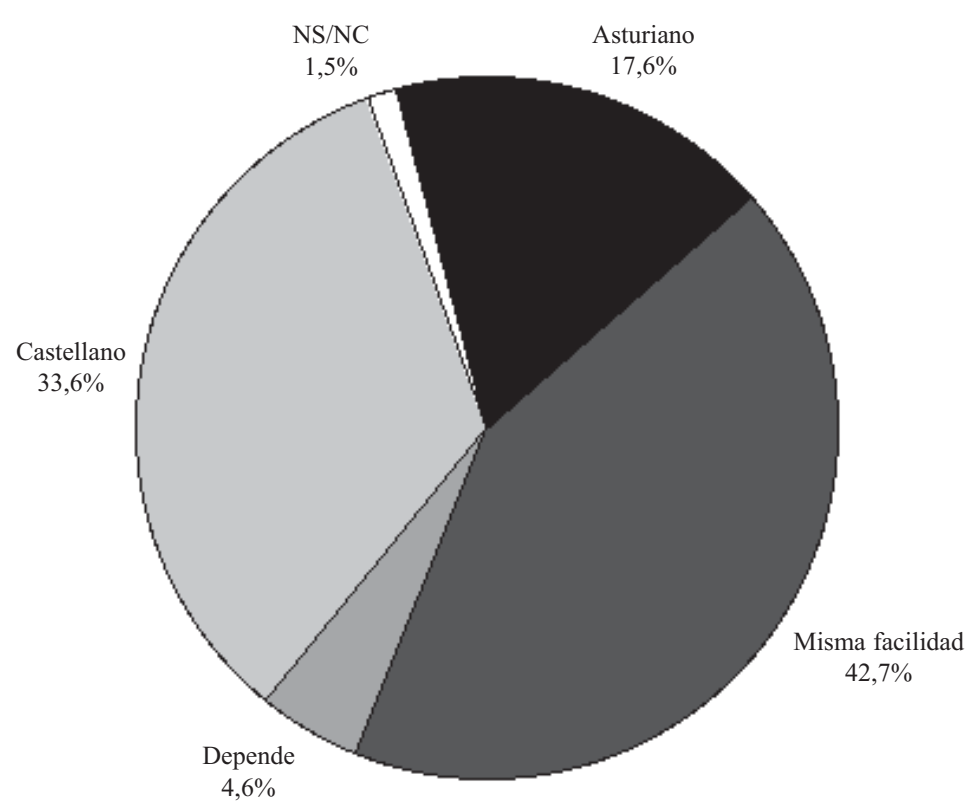

FUENTE: II Estudio sociolingüistico de Asturias - 2002 (en: Llera Ramo 2003: 142).

asturiano con el valor simbólico de la lengua vasca); pero se sitúa por debajo de las restantes comunidades con presencia de más de una lengua (Gráfico 5).

En continuidad con la tendencia observada a lo largo del siglo Xx, parece que la cuestión de la lengua sigue sin encontrarse entre las principales cuestiones políticas de Asturias. El siguiente gráfico muestra los problemas más destacados que los asturianos consideraron de preocupación política principal en el estudio mencionado (Gráfico 6).

\section{Requisitos para la emancipación}

El breve recorrido a través de algunos datos sociológicos indica una serie de dificultades que no permiten fácilmente equiparar la situación del asturiano con la del catalán, del vasco y del gallego. Hemos mencionado el problema de la unidad, de la conciencia de una parte de la población que considera el bable dialecto, el problema de la falta de conocimientos por un gran porcentaje de los asturianos, la consideración de artificialidad del neoasturiano a lo que se añade una 
GRÁFICO 5

Nivel de conocimiento de las lenguas españolas en sus respectivas Comunidades

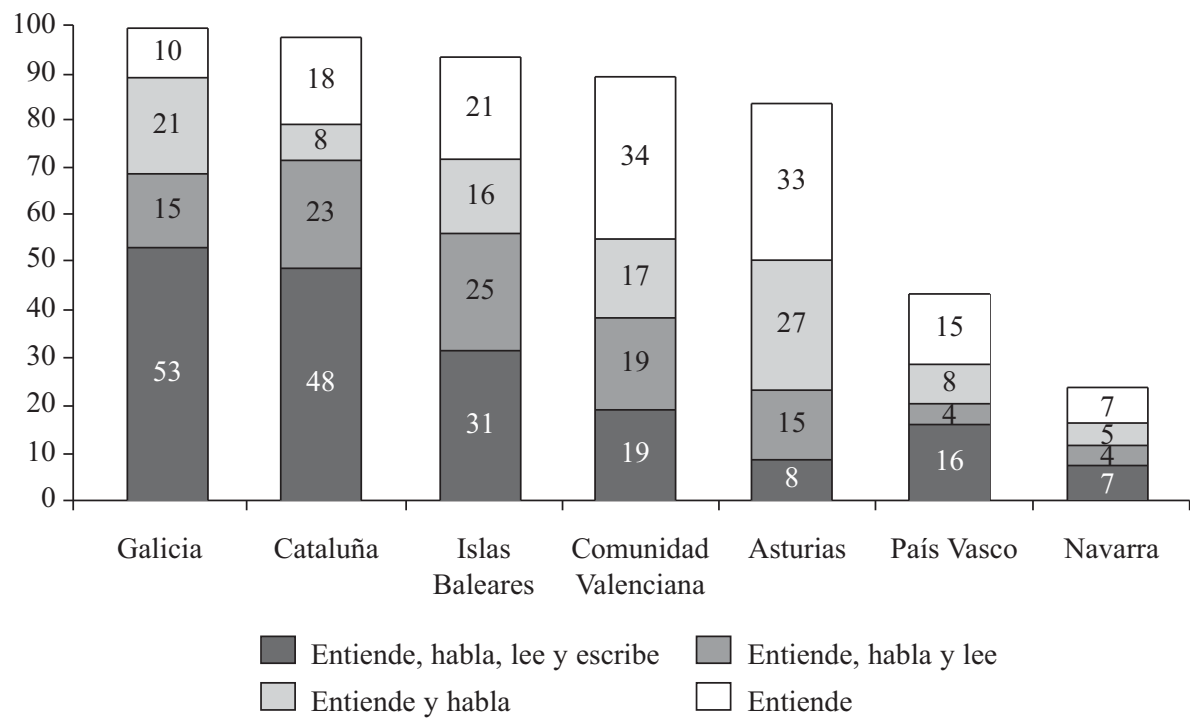

FUENTE: II Estudio sociolingüístico de Asturias - 2002 (en: Llera Ramo 2003: 135).

GRÁFICO 6

Principales problemas de Asturias

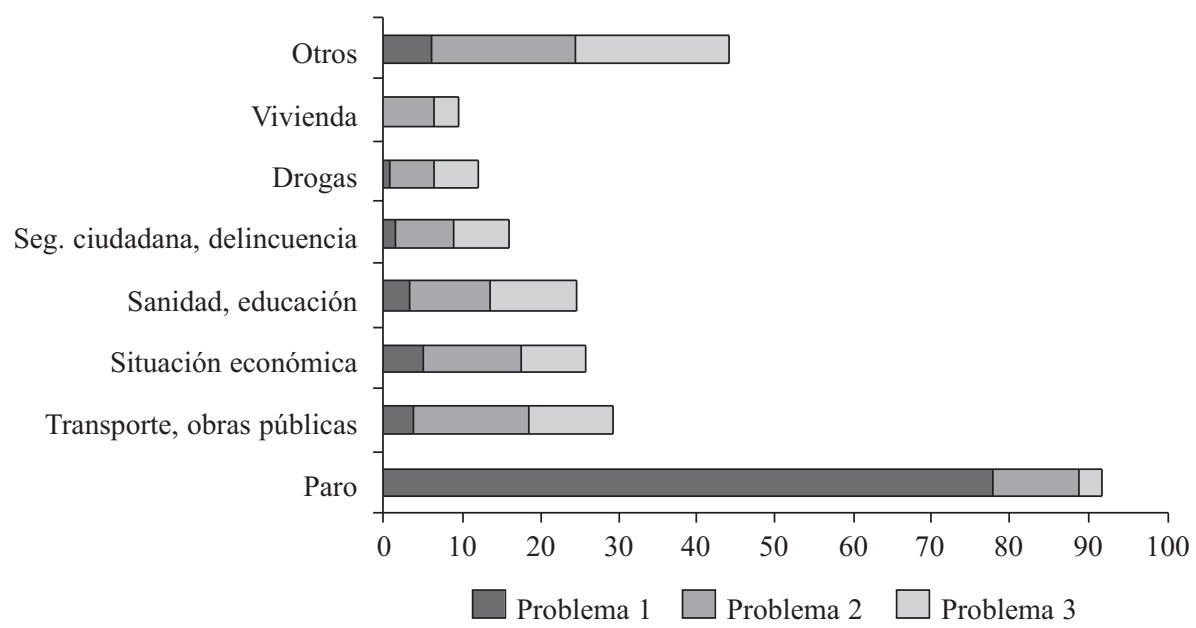

FUENTE: II Estudio sociolingüístico de Asturias - 2002 (en: Llera Ramo 2003: 38). 
extensión reducida de los movimientos urbanos, factores, pues, que solo en parte podrán atribuirse a la política lingüística desde la Transición y que habrá que relacionar también con una situación distinta, no tanto en cuanto a su historia remota y la reivindicación del siglo XIX sino más bien al desarrollo durante el siglo XX. En parte, pues, el discurso asturianista puede adoptar los discursos sobre normalización lingüística generados sobre todo en Cataluña y asimilados por las demás comunidades ya que Asturias cuenta con una base lingüística con un cierto potencial para un proceso de planificación de corpus y estatus, pero en otros aspectos esta base parece estar por debajo del mínimo crítico que se necesita para que tal empresa prometa ser exitosa. Por otro lado, la base es claramente mejor que la que se da en otras situaciones de España, como en el caso del aragonés, donde el potencial lingüístico diferencial es marginal en muchos sentidos (geográfico, generacional, en cuanto al número de hablantes potenciales, etc.).

De lo dicho sobre el mínimo crítico puede deducirse que hay una serie de factores que determinan el potencial de éxito que tiene un movimiento de reivindicación lingüística, factores de los que se puede derivar si más bien es de esperar una ampliación de tal movimiento o si desde la perspectiva actual parece más probable que encuentre dificultades de extensión. Los factores son seguramente múltiples, y no son fáciles de medir, pero la dificultad no implica necesariamente la imposibilidad.

Uno de los factores mencionados es el del problema de identificación de los hablantes tradicionales con la nueva lengua urbana, no idéntica a ningún dialecto particular y, además, fuertemente marcada por la presencia del castellano dada la procedencia de muchos de sus protagonistas de estratos urbanos castellanizados, presencia contra la que reaccionan estos hablantes introduciendo conscientemente formas marcadamente asturianas que, sin embargo, no son compartidas por todos los hablantes tradicionales ya que en parte no pertenecen a sus dialectos y en parte son arcaísmos desaparecidos de las hablas vivas y considerados formas extrañas. La relativa semejanza del neoasturiano hablado con la lengua de contacto y el efecto de extrañamiento que produce frente a las hablas dialectales tradicionales se puede esquematizar de la siguiente manera:

\section{GRÁFICO 7}

Distancia relativa dialectos tradicionales - lengua estándar asturiana - castellano

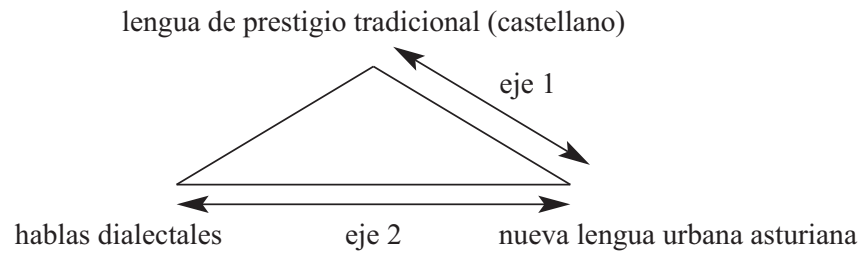


En este triángulo, se compara la distancia de la nueva lengua urbana con la lengua de prestigio tradicional, o sea el español, por un lado, y los dialectos tradicionales, por otro lado. Si la distancia (real o sentida) entre la lengua de prestigio tradicional y la nueva lengua urbana es menor que la que existe entre la lengua urbana y las hablas dialectales tradicionales (es decir, si $1<2$ ), la identificación con la variedad urbana por parte de los hablantes dialectales tradicionales se hace más difícil.

Otro factor es el de la extensión de la lengua común urbana, factor combinable con el prestigio atribuido a esa variedad. El siguiente gráfico intenta esquematizar estos dos factores. La línea vertical representa el grado de extensión relativa de la lengua común, mientras que el eje horizontal representa el prestigio que tiene la variedad en cuestión en la Comunidad. En el caso del catalán, la extensión y el prestigio de la lengua común han llevado a que uno de los discursos más importantes acerca de la lengua catalana es el que observa la nivelación de las variedades dialectales por causa de la influencia de la lengua común ${ }^{14}$, de alto prestigio dentro de la Comunidad catalana (por lo menos en la zona del antiguo Principat). En Galicia, el llamado "gallego común"15 es de creación más

\section{GRÁFICO 8}

Prestigio y extensión relativa de la lengua estándar local en Cataluña, Galicia y Asturias

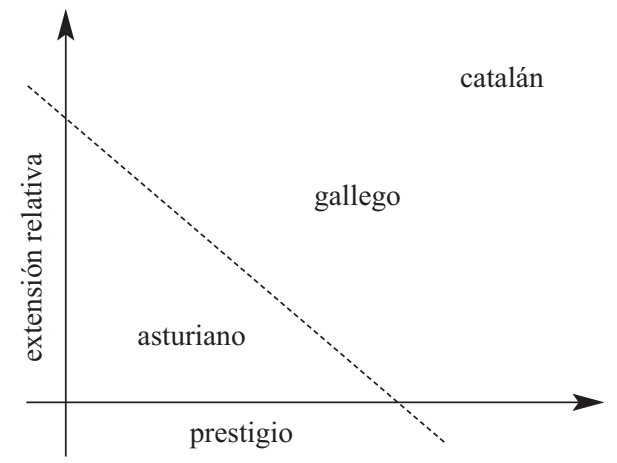

\footnotetext{
14 Véase por ejemplo Massanell i Messalles (2001), Casals i Martorell (2001) y los trabajos presentados en el coloquio Convergència dialectal $i$ estandardització en Tubinga, febrero de 2006 (de próxima publicación, $c f$. <www.kabatek.de/catala $>$ ). En Cataluña, el estándar ya firmemente establecido lleva a otro discurso que en Asturias: no se trata de la cuestión de la aceptación de un fenómeno nuevo sino de la competencia entre dos fenómenos establecidos, las variedades dialectales y el estándar.

15 Cf. Fernández Rei (1991: 30).
} 
reciente pero goza de cierto prestigio y de relativa extensión, ambos factores en aumento en los últimos decenios. En Asturias, tanto la extensión como el prestigio parecen más bien estar, en la actualidad, por debajo de la línea crítica mencionada.

Estos esquemas exigen dos comentarios. En primer lugar, ningún análisis del presente permite hacer predicciones acerca del futuro. Por muy difícil que resulte una "normalización" del asturiano comparable a la del catalán, vasco o gallego, nada dice que sea imposible, solo que los factores que se pueden analizar en el presente apuntan a dificultades mayores que en las otras comunidades. En segundo lugar, todos los factores mencionados son más o menos dinámicos, y altamente dinámicos incluso a corto plazo son los factores de percepción metalingüística y de prestigio de una variedad determinada. En estos factores a corto plazo, una política lingüística tiene mayor posibilidad de influencia. Más arduo es el trabajo de cambio en la competencia real de los hablantes, y la cuestión más importante a la que se tiene que enfrentar un proyecto de creación y de difusión de una lengua diferencial es la del rendimiento individual, económico y de bienestar social que aporta esta "normalización" a los hablantes ${ }^{16}$.

\section{Conclusiones}

Resumiendo, podemos decir, pues, que hay, para la sociología del lenguaje y la cuestión de la emancipación de una lengua minoritaria, una serie de continuos determinantes (distancia, autenticidad, prestigio, número de hablantes, unidad de la lengua, grado de elaboración, etc.). Sintetizando una serie de estos criterios, podemos establecer un continuo de la "salud social" de una modalidad lingüísti$\mathrm{ca}^{17}$. La cuestión de si se trata de una "lengua" o de un "dialecto" no se puede contestar de manera clara (y comprende en parte, además de los factores enumerados, factores políticos e ideológicos) y corresponde, en situaciones dinámicas, muchas veces más a un postulado que a la descripción de la actualidad. Hay lenguas prototípicas y dialectos prototípicos, y el asturiano actual se encuentra a

${ }^{16}$ Hay que señalar que una lengua asturiana estandarizada es algo relativamente nuevo y que no se trata, como se suele decir, de la conservación de la riqueza lingüística tradicional asturiana. La riqueza lingüística tradicional reside en los dialectos asturianos, cuya conservación o pérdida solo indirectamente tiene que ver con la cuestión de la estandarización. No se sabe si la supervivencia de los dialectos es menos o más probable si hay un estándar asturiano, aunque el estándar fomenta el prestigio del asturiano en general y puede afectar así también al prestigio de los dialectos.

17 Es lo que propone analizar Llera Ramo (2003: 12). 
medio camino entre los dos, con la posibilidad -abierta, de momento- de continuar su elaboración y su extensión a largo plazo o la de recaer en el estatus dialectal. La evolución de los últimos decenios apunta a un aumento de los "requisitos para ser lengua", pero solo si encuentra un amplio y duradero apoyo social el "proyecto asturianista" logrará que una lengua asturiana consiga competir de forma continua con el omnipresente castellano. En comparación con otras lenguas de España puede afirmarse que la "salud social" del asturiano es bastante menor que la del gallego, del vasco o del catalán -y pensamos que no es solo por la llamada falta de política lingüística, sino debido a una larga serie de factores, de los que la política lingüística es probablemente más consecuencia que causa.

\section{Bibliografía:}

ACADEMIA DE LA LLINGUA ASTURIANA (2002): Informe sobre la represión y non reconocencia de los drechos llingüisticos n'Asturies. Uviéu, http://www.academiadelallingua.com/pdf/informe_represion.pdf.

BAUSKE, Bernd (1991): "Frühstart als Hemmnis: Anmerkungen zur Rekuperation des Asturianischen und des Galicischen im 19. Jahrhundert", en: Herrmann, Ulfried/Schönberger, Axel (eds.): Studien zur Sprache und Literatur Galiciens. Frankfurt: TFM, 73-87.

- (1995): Sprachplanung des Asturianischen. Die Normierung und Normalisierung einer romanischen Kleinsprache im Spannungsfeld von Linguistik, Literatur und Politik. Berlin: Dr. Köster; version española: Planificación lingüistica del asturiano. La normativización y normalización de una pequeña lengua romance en territorio español desde los puntos de vista lingüistico, literario y político. Trad. por Máximo Martín Serrano (1998). Gijón: vtp.

Bonaparte, Luis Luciano (ed.) (1861): El evangelio de San Mateo traducido al dialecto asturiano de la versión castellana de don Torres Felix [sic] Amat, por un presbitero natural de Asturias; con la cooperación del príncipe Luis Luciano Bonaparte. London: Strangeways \& Walden.

Busto, Xuan Carlos (2002): "Presencia de la llingua asturiana fuera d'Asturies en colecciones documentales ya obres impreses (sieglu XIX)", Revista de Filoloxía Asturiana 2, 97-153.

CASAlS I MARTORELl, Daniel (2001): "Contribució dels media orals a la construcció d’un estàndard de masses plural: presència del dialecte nord-occidental en el llibre d'estil de les emissores radiofòniques de la CCRTV", Zeitschrift für Katalanistik 14, 151-161.

Caveda y Nava, José (1839/1887/1989): Colección de Poesías en dialecto asturiano. Uviéu: Alvizoras.

Coseriu, Eugenio (1981): “Los conceptos de 'dialecto', 'nivel' y 'estilo de lengua' y el sentido propio de la dialectología", Lingüistica española actual III/1, 1-32.

DíAz CASTAÑón, Carmen (1968): "Literatura bable", La estafeta literaria $402-404$ (15IX-1968). 
D’ANDRÉs, Ramón (1995): “La llingua asturiana na sociedá”, en: La llingua asturiana. Uviéu: Academia de la Llingua Asturiana.

Escobar, Francisco (1971): El santo obispo Don Manuel Fernández de Castro. Oviedo. FERNÁNDEZ ReI, Francisco (1991): “A 'questione della lingua' galega”, A Trabe de Ouro 5, 29-40.

Fueru Xulgu. Facsímil del Cod. Hisp. 28 de la Biblioteca del Estado de Baviera. Lectura de Montserrat Tuero Morís. Entamu de Xosé Lluis García Arias, Uviéu 1994.

KABATEK, Johannes (2000): Os falantes como lingüistas. Tradición, innovación e interferencias no galego actual. Vigo: Xerais (Serie Universitaria).

- (2003a): “Estamos dando principio ahora á la gramática asturiana' - Louis Lucien Bonaparte, Manuel Fernández de Castro y la elaboración del asturiano escrito", Actas del I Conceyu Internacional de Lliteratura Asturiana. Uviéu [Oviedo]: Academia de la Llingua Asturiana 2003, 23-51.

- (2003b): “En que consiste o ausbau dunha lingua?”, en: Álvarez de la Granja, María/ González Seoane, Ernesto (eds.): A planificación do léxico galego. Santiago de Compostela: Consello da Cultura Galega/Instituto da Lingua Galega, 37-51.

- (en prensa): Reseña de Llera Ramo/San Martín Antuña 2003, Revista de Filoloxia Asturiana (Uviéu).

KLoss, Heinz (1976): “Abstandsprachen und Ausbausprachen”, en: Göschel, Joachim/ Naid, Norbert/van der Elst, Gaston (eds.): Zur Theorie des Dialekts. Aufsätze aus 100 Jahren Forschung mit biographischen Anmerkungen zu den Autoren. Wiesbaden: Steiner, 301-322.

Llera RAmo, Francisco J. (1991): Los asturianos y la lengua asturiana. Estudio sociolingüistico para Asturias. Uviéu: Serviciu de Publicaciones del Principáu d'Asturies.

Llera Ramo, Francisco J./SAn Martín Antuña, Pablo (2003): II Estudio sociolingüistico de Asturias. 2002. Uviéu: Academia de la Llingua Asturiana.

Massanell i Messalles, Mar (2001): "Morfologia flexiva actual de la Seu d'Urgell i Coll de Nargó: estadis en el procés d'orientalització del català nord-occidental", Zeitschrift für Katalanistik 14, 128-150.

Menéndez Pidal, Ramón (1986[1926]): Orígenes del español. Estado lingüístico de la Península Ibérica hasta el siglo XI. 10a edición (según la tercera muy corregida y adicionada de 1950). Madrid: Espasa Calpe.

Rodríguez Alonso, Alejandro/Feito, José Manuel (eds.) (1997): Manuel Fernández de Castro: Versión asturiana del Dogma de la Inmaculada y Poesía. Uviéu: Alvízoras Llibros.

SÁNCHEZ Vicente, Xuan Xosé (1983): “L’evanxeliu’n bable según San Matéu: una xera de normalización llingüística", Lletres asturianes 9, 20-27.

Vigón, Alfonso (1997): "Prólogu", en: El Evangelio según San Mateo traducido al dialecto asturiano. Facsímil de la edición de 1861. Uviéu: Alvízoras.

WRIGHT, Roger (1989): Latín tardio y romance temprano en España y la Francia carolingia, Madrid: Gredos (versión original: Late Latin and Early Romance in Spain and Carolingian France. Liverpool: Francis Cairns, 1982).

http://constitucion.rediris.es/oapa/codigaut/1981/TCLO07-1981.html http://constitucion.rediris.es/oapa/codigaut/1998/TCLPA01-1998.html 